\title{
Identificación de Struthiopterolichus sp. (Astigmata: Pterolichidae) en avestruces (Struthio camelus camelus) de Cauca, Colombia
}

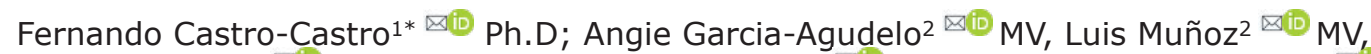

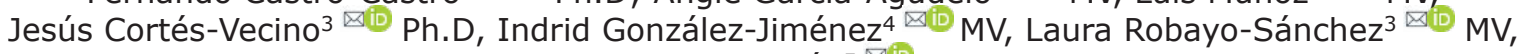 \\ Luis Rivera-Calderón ${ }^{5}$ (1) Ph.D.
}

\begin{abstract}
${ }^{1}$ Universidad Antonio Nariño, Programa de Medicina Veterinaria; Grupo de investigación Quirón, Semillero de investigación en ciencias veterinarias en animales domésticos y silvestres SICAV, Popayán, Colombia.

2 Médico Veterinario particular, Popayán, Colombia.

3Universidad Nacional de Colombia, Facultad de Medicina Veterinaria y de Zootecnia, Laboratorio de Parasitología Veterinaria, Grupo Parasitología Veterinaria, Bogotá D.C., Colombia.

${ }^{4}$ Secretaria Municipal de Salud, Médico Veterinaria, Yumbo, Colombia.

${ }^{5}$ Universidad del Cauca, Facultad de Ciencias de la Salud, Departamento de Morfología; Grupo de Estudios Morfológicos GREMO, Popayán, Colombia
\end{abstract}

*Correspondencia: favian.castro@uan.edu.co

Recibido: Agosto 2020; Aceptado: Diciembre 2020; Publicado: Marzo 2020.

\section{RESUMEN}

Objetivo. Identificar ectoparásitos en las plumas primarias de avestruces adultas alojadas en un criadero del Departamento del Cauca Colombia. Materiales y métodos. Un total de 15 aves adultas (mayores de 7 años), con un peso promedio de $120 \mathrm{~kg}$ (10 hembras y 5 machos) fueron seleccionadas para el estudio. De cada ejemplar fueron extraídas tres plumas primarias del ala, siendo depositadas en bolsas plásticas para ser enviadas al Laboratorio de ciencias básicas de la Universidad Antonio Nariño. Una contramuestra de ácaros fijados en alcohol al $70 \%$ fue enviada a la Universidad Nacional, posteriormente, los parásitos se montaron en láminas examinadas al microscopio. Este estudio fue de tipo descriptivo y a conveniencia. Resultados. Las plumas poseían barbas desorganizadas y en su raquis un polvo de color café, al analizarlas microscópicamente, se obtuvo aproximadamente 1000 especímenes de un ácaro de pluma por muestra, del orden Astigmata, familia Pterolichidae y género Struthiopterolichus sp., lográndose diferenciar hembras, machos, ninfas y larvas. Este es el primer reporte del ácaro de Struthiopterolichus sp. en avestruces de Colombia. Conclusiones. En los avestruces inspeccionados se colectaron plumas que contenían el ácaro Struthiopterolichus sp. La evidencia de este ácaro puede generar alertas de control y vigilancia sanitaria al interior del criadero.

Palabras claves: Acari; aves; ectoparásitos; plumas; Struthioniformes (Fuentes: DeCS, FAO, MeSH).

\section{ABSTRACT}

Objetive. Identify ectoparasites in the primary feathers of adult ostriches in a hatchery of Department from Cauca, Colombia. Materials and methods. A total of fifteen adult animals (over 7 years old) with an average weight of $120 \mathrm{~kg}$ (10 were females and 5 males) were selected for the study. From

Como citar (Vancouver).

Castro-Castro FF, Garcia-Agudelo AD, Muñoz LM, Cortés-Vecino JA, González-Jiménez IM, Robayo-Sanchez LN, Rivera-Calderón LG. Identificación de Struthiopterolichus sp. (Astigmata: Pterolichidae) en avestruces (Struthio camelus camelus) de Cauca, Colombia. Rev MVZ Córdoba. 2021; 26(2):e2154. https://doi.org/10.21897/rmvz.2154 
each specimen, three primary feathers were extracted in the wing, being deposited in plastic bags and sent to the Basis science laboratory at Universidad Antonio Nariño. A counter sample of mites fixed in alcohol at $70 \%$ was sent to laboratory of the Universidad Nacional, then, the parasites were mounted o slides and under the microscope. This study was descriptive and convenient. Results. The feathers had disorganized barbs and a brown powder on the rachis. Microscopically was identified approximately 1000 specimens of a feather mite per sample of order Astigmata, family Pterolichidae and genus Struthiopterolichus sp., being able to differentiate male, female, nymphs and larvae. This is the first report of the mite Struthiopterolichus sp. in ostriches from Colombia Conclusions. In the ostriches inspected were collected feathers that contained the mite Struthiopterolichus sp. The evidence of this mite can generate alerts of control and health surveillance inside of the hatchery.

Keywords: Acari; birds; ectoparasites; feathers; Struthioniformes (Sources: DeCS, FAO, MeSH).

\section{INTRODUCCIÓN}

El avestruz (Struthio camelus) es una especie de ave corredora originaria del continente africano que ha sido domesticado para fines productivos. Dentro de la industria del ejemplar son relevantes tres productos: carne, cuero y plumas; la carne es rica en proteínas y baja en grasas, mientras que el cuero y el plumaje son utilizados ampliamente en la industria de la moda $(1,2,3)$. Los productos del animal también se implementan para decoración y la limpieza de máquinas y equipos finos (4).

Esta ave es muy resistente, posee alta capacidad de adaptación a varias condiciones nutricionales y climáticas lo que le ha permitido distribuirse en varios continentes incluyendo América. En Colombia, llegó en la década de los 90 y actualmente la industria se encuentra bien establecida en los departamentos del Meta, Cundinamarca, Cauca, Valle del Cauca, Boyacá y Quindío (5).

El Instituto Colombiano Agropecuario mantiene una estricta vigilancia y control en los criaderos autorizados en el país, evaluando su bioseguridad y plan sanitario (donde se exige la vacunación contra Newcastle). Sin embargo, a pesar de las medidas sanitarias para evitar la introducción y transporte de avestruces con posibles agentes patógenos, ya se han identificado algunos criaderos con parásitos internos que pueden desencadenar enfermedades en animales susceptibles $(5,6)$.

Por otro lado, se ha descrito por diferentes autores que los ratite también pueden portar algunos ectoparásitos en su piel y el plumaje, entre estos sobresalen los ácaros (Struthiopterolichus bicaudatus y Dermoglyphus pachycnemis) $(7,8,9,10)$. Algunos de los parásitos mencionados pueden causar prurito e irritación en la piel, así como daño y caída de plumas, lo que representa menor bienestar para el ave y baja calidad del producto final $(4,7,10,11)$. Por lo anterior, el objetivo principal de este estudio fue identificar ectoparásitos en las plumas primarias de los especímenes adultos en un criadero del departamento del Cauca.

\section{MATERIALES Y MÉTODOS}

Área de estudio. El estudio se realizó en un criadero de avestruces localizado en la zona rural del municipio de Puerto Tejada en el norte del Departamento del Cauca, Colombia, con coordenadas $3^{\circ} 13^{\prime} 48^{\prime \prime} \mathrm{N}, 76^{\circ} 25^{\prime} 3^{\prime \prime} \mathrm{O}$. Este se localiza a una altitud aproximada de 970 m.s.n.m, con temperatura media de $24^{\circ} \mathrm{C}$, precipitación pluvial entre 1000 a 2000 mm y una humedad relativa del 70 al $90 \%$. Los animales del estudio se distribuyeron en corrales clasificados de acuerdo a la edad y estado reproductivo, siendo alimentados con ración y diversas especies de plantas como nacedero (Trichanthera gigantea), king grass (Pennisetum purpureum), maní forrajero (Arachis pintoi) y matarratón (Gliricidia sepium).

Animales de estudio. El criadero visitado contaba con 290 avestruces de diferentes edades de los cuales 200 eran adultos. Este estudio fue de tipo descriptivo y a conveniencia, se seleccionaron a conveniencia 15 avestruces adultos de cuello rojo (Struthio camelus camelus), mayores de siete años con peso promedio de $120 \mathrm{~kg}$. De éstos, diez eran hembras y cinco, machos. El trabajo tuvo una duración de dos meses entre la colecta y los resultados obtenidos.

Manejo y muestreo. El presente estudio fue avalado por el comité de ética de la Universidad Antonio Nariño (número de protocolo 2019-02). 
El manejo de las aves fue ejecutado con la ayuda de personal calificado. Para la captura, se empleó una vara de metal, forrada con plástico que sujetaba el cuello del avestruz, seguidamente se cubrió la cabeza del animal para evitar mayor estrés. El procedimiento para la obtención del plumaje tuvo un promedio de cinco minutos por ave. De cada ejemplar se extrajeron tres plumas primarias del ala, las cuales fueron depositadas en bolsas plásticas, rotuladas y enviadas al laboratorio de Ciencias Básicas de la Universidad Antonio Nariño (UAN), Popayán, Colombia.

Análisis de laboratorio. Se logró identificar que todas las plumas colectadas estaban infestadas por ácaros a través de un microscopio óptico (Leica DM300) con un objetivo de 10 y 40x. Se envió una contramuestra con estos especímenes conservados en alcohol al $70 \%$ al Laboratorio de Parasitología Veterinaria de la Universidad Nacional de Colombia (UNAL). Los ácaros fueron clarificados y montados en láminas con solución de Hoyer's para su posterior análisis parasitológico, la conservación, clarificación y montaje se efectuaron de acuerdo con la metodología de Faccini et al (12). El diagnóstico se realizó usando un microscopio óptico (Nikon 200 Eclipse, Tokio, Japón) para su respectiva clasificación.

\section{RESULTADOS}

Todas las plumas primarias de los avestruces analizados estaban infestadas por ácaros. Se obtuvo material suficiente en el raquis (Figura 1) para la posterior identificación taxonómica. En el Laboratorio de Parasitología Veterinaria de la Universidad Nacional de Colombia se diagnosticó la presencia de un ácaro de pluma del género Struthiopterolichus (Astigmata:Pterolichidae), con alta probabilidad de pertenecer a la especie $S$. bicaudatus. En los campos del microscopio se observaron estadios adultos (Figuras $2 \mathrm{~A}$ y 2B), así como, de ninfa y larva (Figuras $3 \mathrm{~A}$ y 3B). Estos especímenes fueron depositados en la Colección Parasitológica Veterinaria CPV-UN con registro CPV-UN (A6).
Los ácaros fueron caracterizados morfológicamente por un dimorfismo sexual evidente desde el objetivo de menor aumento (4x) en microscopía de luz. Macho (Figura 2A): Largo total (LT) 600 $\mu \mathrm{m}$, presencia de una placa anterior (pa) y una posterior $(\mathrm{pp})$ que presenta un patrón poligonal desde la vista dorsal, lóbulos posteriores (Ip) con ventosas ( $v$ ), bifurcación (b) de los lóbulos posteriores y dos setas largas en cada lóbulo. Hembra (Figura 2B): LT 800 $\mu \mathrm{m}$, presencia de una placa anterior ( $p a)$ y una posterior ( $p p$ ) que presenta un patrón poligonal desde la vista dorsal, (pg) poro genital y 4 setas largas en el borde posterior sin bifurcación del idiosoma. Ninfa (Figura 3A): LT $\sim 600 \mu \mathrm{m}$, presencia de una placa anterior (pa) y una posterior ( $p p$ ) que presenta un patrón poligonal desde la vista dorsal y 4 setas largas en el borde posterior del idiosoma. Larva (Figura 3B): LT 300 $\mu \mathrm{m}$, presencia de tres pares de patas, una placa anterior ( $\mathrm{pa}$ ) y una posterior ( $\mathrm{pp}$ ) que presenta un patrón poligonal desde la vista dorsal y 2 setas largas en el borde posterior del idiosoma. Adicionalmente, existe similitud del tamaño en todos los pares de patas en los diferentes estadios de desarrollo $(13,14)$.

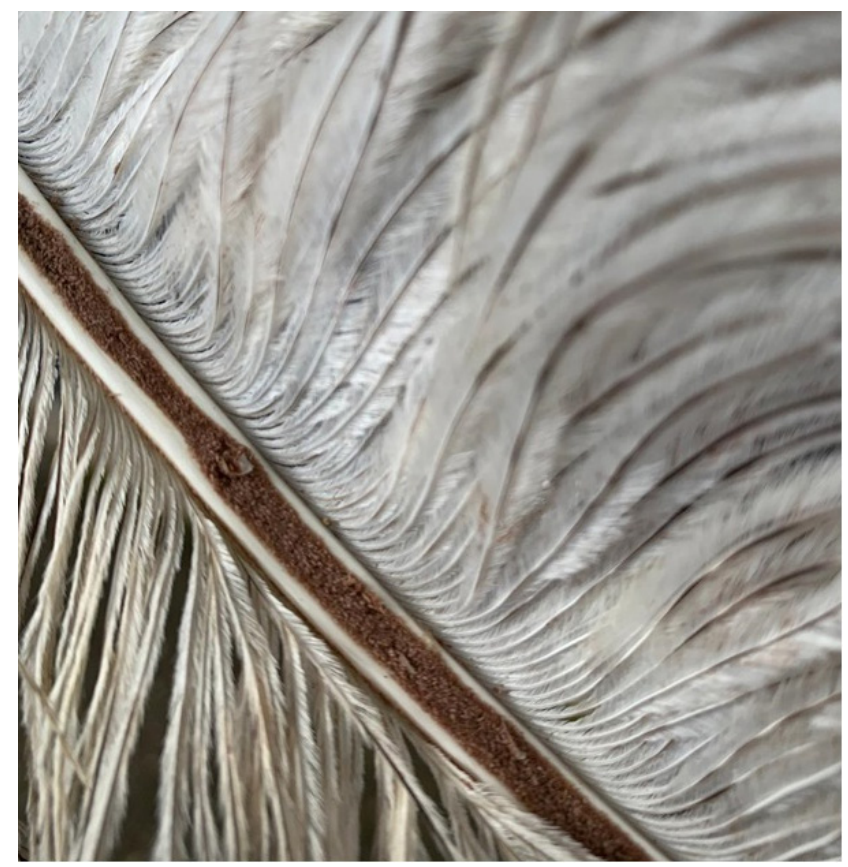

Figura 1. Presencia de ácaros con aspecto de "polvo color café" en el raquis de la pluma. 

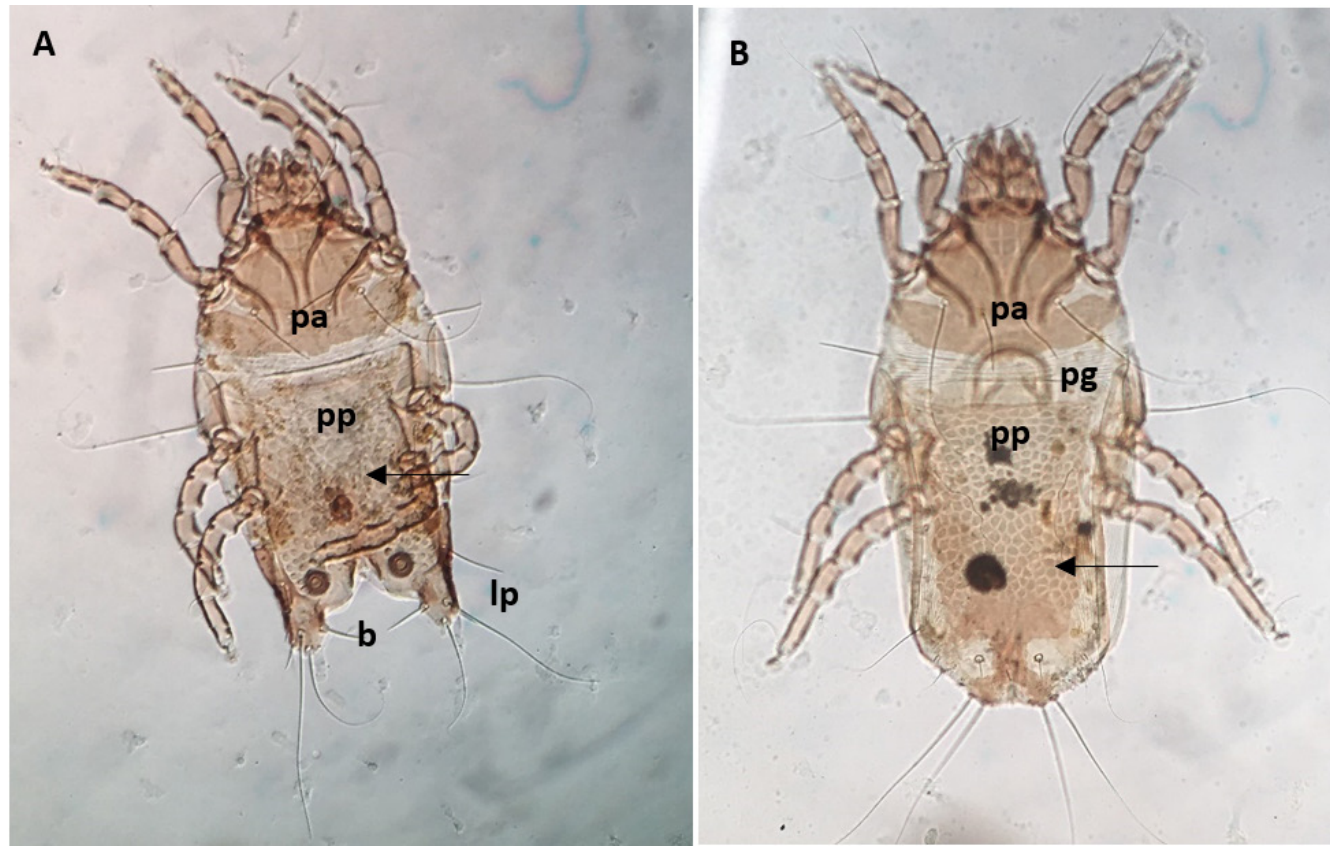

Figura 2. Struthiopterolichus sp. A. Macho (10x), pa (placa anterior), pp (placa posterior), Ip (lóbulos posteriores), b (bifurcación), v (ventosa), flecha (patrón poligonal). B. Hembra (10x), pa (placa anterior), pp (placa posterior), pg (placa genital), flecha (patrón poligonal).

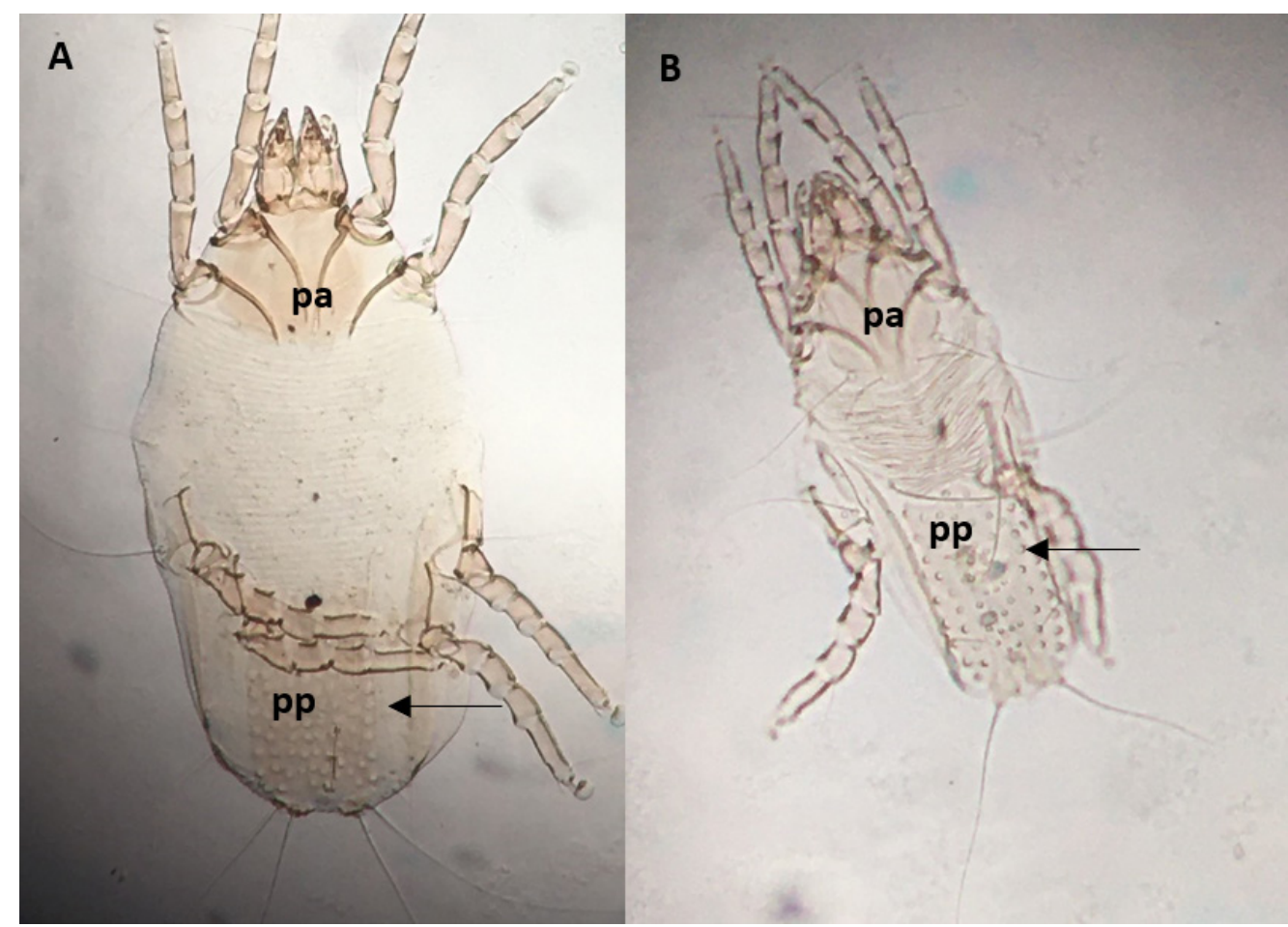

Figura 3. Struthiopterolichus sp. A. Ninfa (10x), pa (placa anterior), pp (placa posterior, flecha (patrón poligonal). B. Larva (10x), pa (placa anterior), pp (placa posterior), flecha (patrón poligonal).

\section{DISCUSIÓN}

En este estudio, se observó que el criadero de avestruces ubicado en el departamento del Cauca, Colombia, presentaba infestaciones de ácaros del género Struthiopterolichus sp., localizados en el raquis de las plumas primarias. Estos ácaros se conocen popularmente como ácaros de las plumas y fueron descritos por primera vez en el año 1843 en París en el avestruz (15). Sin embargo, desde el punto de vista taxonómico, se ha constituido en una 
dificultad la identificación, ya que no existen claves taxonómicas específicas y el ácaro se han reportado en anteriores estudios como Gabucinia bicaudata $(4,7,10)$, Pterolichus bicaudatus y Struthiopterolichus bicaudatus $(12,16)$. La nomenclatura de este último hace referencia a su relación con el hospedero (Struthio camelus), ya que es considerado un ácaro especieespecífico, por lo cual, estaría en concordancia para este primer reporte. Pero debe tenerse en cuenta que, aunque la descripción morfológica aquí presentada y la evidencia de la literatura sugieren que se trata de esta especie, es importante poder obtener secuencias genéticas que permitan relacionarlo con otras especies de la familia Pterolichidae.

En el trabajo actual también se evidenció mal estado de las plumas de las aves seleccionadas (falta de brillo y poca organización en las barbas), posiblemente, debido a la presencia del ácaro. Hallazgos similares fueron descritos por Faccini y colaboradores en un criadero ubicado en el estado de Minas Gerais, Brasil. Estos autores identificaron plumas rotas, con barbas desorganizadas o ausentes y prurito en un total 285 ejemplares mayores de 13 meses; en las plumas de esas aves se identificó el ácaro $S$. bicaudatus (12).

El ácaro de las plumas se ha reportado en diferentes países del mundo, en algunos casos dependiendo del grado de infestación se indican signos clínicos como pérdida de las plumas y reducción de la calidad de la piel $(7,16,17)$. A pesar de lo anterior, aún es controversial la importancia económica del $S$. bicaudatus en la industria del avestruz. En otros ratites como los ñandúes (Rhea americana) se han reportado ectoparásitos como el Struthiolipeurus nandu, los cuales presentan implicaciones con pérdidas económicas; este piojo se aloja principalmente en las alas del hospedero, y retarda su crecimiento (18).
Por su parte, faltan estudios epidemiológicos para determinar si se puede compartir $S$. bicaudatus con otras aves, por ejemplo, aves de corral o de vida silvestre que tengan una cercanía a las producciones de avestruces (14). Después de realizar el diagnóstico del ácaro, se recomendaron tratamientos con ivermectina $u$ organofosforados en los avestruces adultos con las dosis sugeridas por otros autores $(7,10)$. No obstante, también se le mencionó al propietario la importancia de la rotación de estos acaricidas con el fin de evitar resistencia parasitaria, la cual se ha tornado cada vez más frecuente en los últimos 30 años $(19,20)$. En este estudio no se realizó ninguna inspección en las aves menores de 7 años y en juveniles (menores de 3 años) porque no fue concedida la autorización por parte del administrador para el muestreo, además en el momento del estudio existían pocas aves en esos grupos etarios.

Este estudio es el primer reporte de esta especie de ácaro en avestruces en Colombia, que llevará a ahondar en el diagnóstico molecular, obtención de secuencias genéticas y consolidación de claves taxonómicas que faciliten la clasificación de estos tipos de ácaros.

Futuros estudios son necesarios para la evaluación del ciclo biológico del ácaro, abarcando diferentes grupos etarios del ave (para verificar presencia del ácaro o constatar que solo puede afectar a adultos). Del mismo modo, estos estudios ayudarán a crear estrategias de control y manejo adecuado en las producciones de avestruces.

\section{Conflicto de intereses}

Los autores de este estudio manifiestan que no existe conflicto de intereses con la publicación de este manuscrito.

\section{REFERENCIAS}

1. Brassó LD, Béri B, Komlósi I. Studies on Ostrich (Struthio Camelus) - Review. Acta Agraria Debreceniensis. 2020; 1:15-22. https://doi.org/10.34101/actaagrar/1/3772

2. Brand, TS, Kritzinger, WJ, Van der Merwe, DA, Muller, A., Hoffman, LC y Niemann, GJ. Feather and skin development of ostriches Struthio camelus. J S Afr Vet Assoc. 2018; 89. https://dx.doi.org/10.4102/jsava. v89i0.1556
3. Brand TS, Jordaan JW. The contribution of the South African ostrich industry to the national economy. Appl Anim Husb Rural Develop. $2011 ; 4(1): 1-7$. https://pdfs. semanticscholar.org/39c4/50b634ad985a7 8132da6d5f4886dd8b49c9b.pdf

4. Elsayed NM. Occurrence of some Parasites in farmed ostriches (Struthio camelus) in Egypt. EVMSPJ. 2016; 6(12):91-100. http:// dx.doi.org/10.21608/evmspj.2016.37345 
5. Mariño GA, Ramírez A, Cortés JA. Libyostrongylus douglassii (Strongylida: Trichostrongylidae) in ostrich (Struthio camelus) farms from Colombia. Vet Parasitol. 2017; 235:53-56. http://dx.doi. org/10.1016/j.vetpar.2017.01.007

6. Paz J, Rivera L, Tigreros S, Acosta A, Sarmiento J, Valencia C, Castro F. Identificación de parásitos gastrointestinales en un criadero de avestruces (Struthio camelus) del departamento del Cauca. Rev Colombiana Cienc Animal RECIA. 2019; 11(1):e698. https://dx.doi.org/10.24188/ recia.v11.n1.2019.698

7. Mohammed BR, Malang SK. Common Ectoparasites of Ostrich (Struthio camelus) and their Control-A review. Res J Animal Veterinary and Fishery Sci. 2015; 3(10):2329. http://www.isca.in/AVFS/Archive/v3/ i10/3.ISCA-RJAVFS-2015-023.pdf

8. Cooper RG, Doumani HAA. The presence of quill mites (Gabucini bicaudata) and lice (Struthiolipeurus struthionis) in ostrich wing feathers. JI S Afr vet Ass. 2006; 77(1): 9-11. https://dx.doi.org/10.4102/jsava. v77i1.332

9. Ponce-Gordo F, Herrera S, Castro AT, García-Durán B, Martínez-Díaz RA. Parasites from farmed ostriches (Struthio camelus) and rheas (Rhea americana) in Europe. Vet Parasitol. 2002; 107:137160. https://dx.doi.org/10.1016/S03044017(02)00104-8

10. Nemejc K, Lukesova D. Parasite Fauna of Ostriches, Emus and Rheas. Agric trop subtrop. $2012 ; 45(1): 45-50$. https://dx.doi. org/10.2478/v10295-012-0007-6

11. Smith DA. Palaeognathae: Apterygiformes, Casuariiformes, Rheiformes, Struthioniformes; Tinamiformes. Pathology of Wildlife and Zoo Animals. 2018; 22(1):635651. http://dx.doi.org/10.1016/B978-0-12805306-5.00026-2

12. Faccini JLH, Verocai GG, Lopes LN, Souza CP. Occurence of Struthiopterolichus bicaudatus (Acari; Pterolichidae) in southeastern Brazil. Arq Bras Med Vet Zootec. 2006; 58(5):959960. http://dx.doi.org/10.1590/S0102$\underline{09352006000500039}$
13. Proctor HC. Feather mites (Acari: Astigmata): ecology, behavior, and evolution. Annu Rev Entomol. 2003; 48(1):185209. https://dx/doi/10.1146/annurev. ento.48.091801.112725

14. Lima MM, Carvalho E, Soares EE, Hernandez A. Feather mites (Acari: Astigmata) of the Zoobotanical State Park in Teresina, Brazil. Acarologia. 2019; 59(4):424-432. http:// dx.doi.org/10.24349/acarologia/20194345

15. André M. Sarcoptides plumicoles parasites des autruches. Acarologia. 1960; 2:556567.

16. Halliday RB. Struthiopterolichus bicaudatus (Gervais) (Acari:Pterolichidae): A feather mite pest of the ostrich in Australia. Wildlife Zoos. 2006; 84(1-2):68-69. https://dx.doi. org/10.1111/j.1751-0813.2006.tb13133.x

17. Kummrow MS. Ratites or Struthioniformes Struthiones, Rheae, Cassuarri, Apteryges (Ostriches, Rheas, Emus, Cassowaries, and Kiwis), and Tinamiformes (Tinamous). In: Miller E, Fowler ME, Editors. Flowler's Zoo and Wild Animal Medicine. 8th Edition. Saunders; 2015. https://dx.doi.org/10.1016/B978-14557-7397-8.00009-8

18. Gallo SS, Ederli NB, Oliveira FC. Endoparasites and ectoparasites of rheas (Rhea americana) from South America. Trop Biomed. 2018; 35(3):684-695. http://msptm.org/files/ Vol35No3/684-695-Gallo-SSM.pdf

19. McNair CM. Ectoparasites of medical and veterinary importance: drug resistance and the need for alternative control methods. J Pharm Pharmacol. 2015; 67(3):351-363. https://dx.doi.org/10.1111/jphp.12368

20. Doherty E, Burgess S, Mitchell S, Wall R. First evidence of resistance to macrocyclic lactones in Psoroptes ovis sheep scab mites in UK. Vet Rec. 2018; 182. http://dx.doi. org/10.1136/vr.104657 